\title{
Physical Model of the Pulmonary Circulation Designed for Investigation on Cardiac Output Measurement by Means of the Thermodilution Method
}

\author{
M. Gawlikowski* And T. Pustelny \\ Faculty of Electrical Engineering, Silesian Technical University, Gliwice, Poland
}

The "gold clinical standard" of cardiac output measurements is the thermodilution method, implemented by the Swan-Ganz catheter. The unreliability of obtained results is difficult to estimate experimentally, due to the lack of reference methods or the risk of serious interferences in the measured object. Moreover, the pulsating blood flow produced by the heart is difficult to measure. Therefore it is advisable to develop the physical model of the pulmonary circulation, designed to investigate the accuracy of cardiac output measurements by means of the thermodilution method.

PACS: 87.19.Hh, 47.63.Ec, 82.60.Fa

\section{Introduction}

Nowadays the "gold clinical standard" of the hemodynamic parameters measurement is pulmonary artery catheterization (PAC) by means of the Swan-Ganz catheter $[1,2]$. For more than 40 years the cardiac output $(\mathrm{CO})$ has been measured by means of the thermodilution method [2]. In spite of numerous disadvantages connected with invasiveness and high susceptibility to disturbances the PAC and thermodilution techniques are the most fundamental methods of the hemodynamics parameters measurements [3].

The estimation of the unreliability of $\mathrm{CO}$ measurement by means of the thermodilution method is complicated because of the following reasons:

a) the lack of other reference methods with suitable high accuracy $[3,4]$,

b) the significant influence of reference method on examined object (human or experimental animal),

c) the pulsating character of blood flow in the circulatory system [5].

Many authors compare the results of $\mathrm{CO}$ measurements obtained by means of the thermodilution and the Fick methods [6-14] but in fact, the accuracies of the others methods are unknown. Therefore only the correlation of results may be estimated. The accuracy of thermodilution may be determined theoretically $[3,4]$ by analysis of the mathematical model of dilution (e.g. the Stewart-Hamilton equation [1,3] or the local diffusion random walk model $[9]$ ). In those cases some physical

* corresponding author; e-mail:

maciej.gawlikowski@poczta.onet.pl phenomena (difficult to mathematical definition) may be omitted and conclusions about real unreliability of the method may be wrong.

\section{Goal}

Comprehensive investigations of unreliability of the thermodilution method may be performed by means of the physical modeling of the equivalent parts of circulatory system [15-19]. The goal of this work was to develop the physical model of the pulmonary circulation, designed for the investigations of $\mathrm{CO}$ measurements by means of the thermodilution method.

\section{Material and methods}

The developed physical model of the pulmonary circulation consists of the following blocks: the heart simulator, the flow measurement system, and the supplying and control system. The detailed discussion of mentioned block is described below.

\subsection{The heart simulator}

The developed model simulates the part of the pulmonary circulation (Fig. 1.). It consists of the physical models of the right atrium, the right ventricle, the pulmonary artery valve, the tricuspid valve, the pulmonary trunk, and the left and right pulmonary arteries. The spatial arrangement of these structures is similar like in the biological heart. The flexible membranes of ventricle and atrium were made of polyurethane by means of dipping method (the glass moulds were utilized). The polyurethane, tri-leaflet valves, originating with the models of the ventricular assist devices, were applied. The model allows simulating various diameters of the pulmonary vessels. The hydrodynamic load was a hydrostatic pressure generated by constant height of the column of operating liquid. The detailed geometrical features were presented in Table I. 
The comparison of the parameters of physical model and reference.

TABLE I

\begin{tabular}{|c|c|c|}
\hline Feature & Value obtained in model & Reference \\
\hline $\begin{array}{l}\text { anatomy: } \\
\text { volume of right atrium } \\
\text { volume of right ventricle } \\
\text { diameter of RPA } \\
\text { diameter of LPA } \\
\text { diameter of PT }\end{array}$ & $\begin{array}{l}30 \mathrm{ml} \\
110 \mathrm{ml} \\
1.3 \mathrm{~cm} \\
1.0 \mathrm{~cm} \\
2.6 \mathrm{~cm}\end{array}$ & $\begin{array}{c}20 \ldots 40 \mathrm{ml} \\
100 \ldots 160 \mathrm{ml}(\mathrm{RVEDV}) \\
1.2-2.4 \mathrm{~cm} \\
0.9-2.1 \mathrm{~cm} \\
1.5-2.9 \mathrm{~cm}\end{array}$ \\
\hline $\begin{array}{l}\text { hemodynamics: } \\
\text { heart rate } \\
\text { cardiac output } \\
\text { ejection fraction } \\
\text { blood flow velocity in PT }\end{array}$ & $\begin{array}{c}30 \ldots 120 \mathrm{BPM} \\
\max .4 .5 \mathrm{~L} / \mathrm{min} \\
\max .80 \% \\
0.95 \mathrm{~m} / \mathrm{s} \\
\end{array}$ & $\begin{array}{c}50 \ldots 100 \mathrm{BPM} \\
\text { phys. } 4-8, \text { pat. }<3.5 \mathrm{~L} / \mathrm{min} \\
40-60 \% \text { for } \mathrm{RV} \\
0.6-0.9 \mathrm{~m} / \mathrm{s}\end{array}$ \\
\hline $\begin{array}{l}\text { thermodynamics: } \\
\text { specific heat of blood } \\
\text { mass density of blood } \\
\text { temperature of blood }\end{array}$ & $\begin{array}{c}3528 \mathrm{~J} /(\mathrm{kg} \mathrm{K}) \\
1065 \mathrm{~kg} / \mathrm{m}^{3} \\
T_{\mathrm{amb}} \ldots 42^{\circ} \mathrm{C} \\
\end{array}$ & $\begin{array}{c}\text { typ. } 3500 \mathrm{~J} /(\mathrm{kg} \mathrm{K}) \\
\text { typ. } 1055 \mathrm{~kg} / \mathrm{m}^{3} \\
\text { from hypothermia to } 41.5^{\circ} \mathrm{C}\end{array}$ \\
\hline $\begin{array}{l}\text { pathology: } \\
\text { diseases of the valves } \\
\text { arrhythmia }\end{array}$ & $\begin{array}{r}\text { tricuspid and pulmon } \\
\text { possil }\end{array}$ & $\begin{array}{l}\text { artery valves regurgitation } \\
\text { to simulate }\end{array}$ \\
\hline
\end{tabular}

The model which produces pulsating flow is divided into ejection (systole) and suction (diastole) phases. The contraction of the atrium and ventricle are independent of each other. It is possible to independently set the atrium contraction time, the ventricle contraction time and the diastole time. The heart model is able to simulate the arrhythmia and the dysfunction of tricuspid and pulmonary artery valves. The operating liquid is a blood substitute with similar thermodynamic parameters $(30 \%$ glycerin and water solution with antibacterial agent). The injecting of indicator $(0.9 \% \mathrm{NaCl})$ may change those parameters, therefore the mass density of the mixture is measured by means of the glass aerometer. The modification of operation liquid concentration allows the modeling of thermodynamic parameters of blood. The temperature of medium is stabilized by electronic regulator to an accuracy of $+0.1^{\circ} \mathrm{C} /-0.3^{\circ} \mathrm{C}$; therefore, it is possible to simulate the states of hypothermia and fever. Due to the close hydraulic character of developed model it allows us to simulate the recirculation of the indicator.

\subsection{The flow measurement system}

The measurement of pulsating flows is rather complicated [5]. The accuracy of modern technical solutions (e.g. ultrasound flow meters) is poor and reaches about 10\%. In developed system (see Fig. 2 and Fig. 3) the pulsating flow, which is produced by the heart simulator, is transformed to the continuous flow in the subsystem consisting of a centrifugal pump and an equalizing tank. In steady-state the pulsating flux flowing into the tank equals the continuous flux flowing through the centrifugal pump. In consequence the level of liquid inside

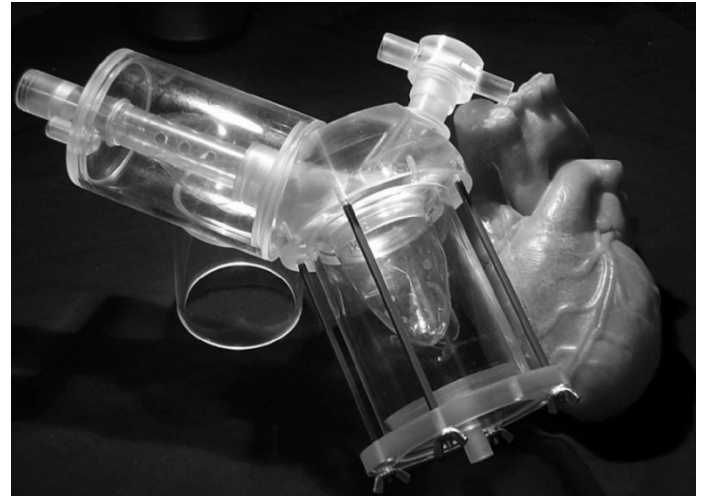

Fig. 1. The physical model of the heart.

the equalizing tank is constant. To measure only the pulsating flow, produced by the heart simulator, the levels of liquid into the reservoir and equalizing tank must be the same. This state may be achieved by setting the rotational speed of the centrifugal pump: automatically (by means of a regulator based on measurement of the hydrostatic pressure difference in those vessels) or manually. The continuous flow (which — in steady-state - equals the pulsating flow) is measured with accuracy of $1.5 \%$ by a calibrated rotameter.

\subsection{The supplying and control system}

The model of the heart is pneumatically supplying by means of a pneumatic generator. The pneumatic wave for supplying the atrium and the ventricle is generated by electrically controlled valves driving by means of NI- 


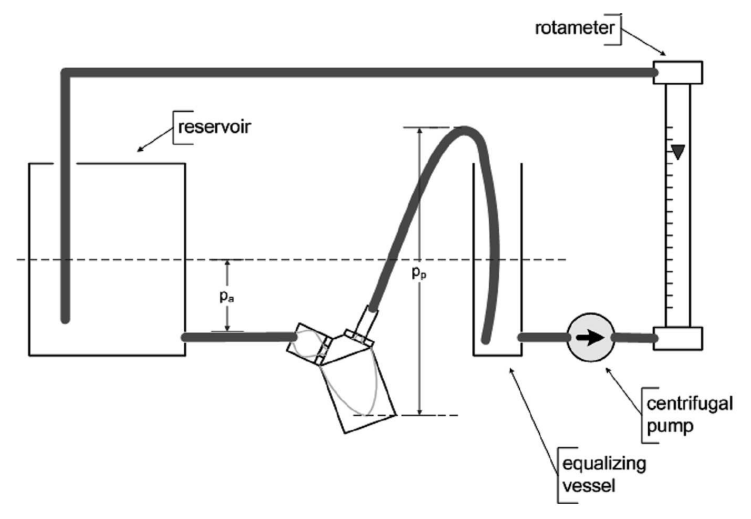

Fig. 2. The scheme of the model of pulmonary circulation and flow measurement system.

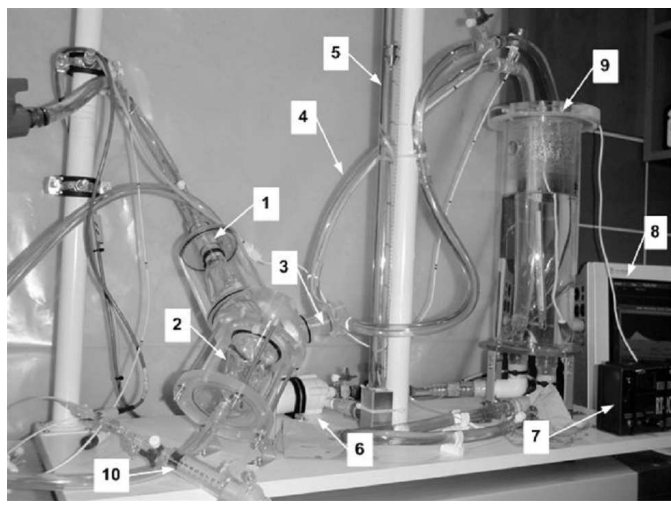

Fig. 3. The physical model of pulmonary circulation designed for cardiac output measurement by means of the thermodilution method. 1 - atrium, 2 - ventricle, 3 - pulmonary trunk, 4 - pulmonary artery, 5 - rotameter, 6 - centrifugal pump, 7 - temperature regulator, 8 - patient monitor, 9 - equivalence tank, 10 - syringe for injection of the indicator.

-USB-6221 the multifunction data acquisition device and LabVIEW software.

\subsection{The verification of the model operation correctness}

The verification of the developed model was performed by means of:

a) comparison of selected functional parameters of the model with the reference (taken from the medical literature or non-invasive examinations of the patients),

b) comparison of course of the dilution phenomenon occurring in the physical model and the human circulatory system.

To measure the diameters of the pulmonary vessels and the velocity of blood flow the transthoracic echocardiography examinations were performed. The pulsating flow of the operation liquid into the model was measured by means of ultrasound flowmeter T206 and 14C probe (Transonic, accuracy $\pm 8 \%$ ). The indicator dilution curve (IDC) was measured during the invasive cardiac output examination (Hemodynamics Lab., Specialized Hospital in Zabrze, Poland) by means of the room temperature thermodilution and the Swan-Ganz catheter (7F size).

\section{Results}

Three patients were examined by means of transthoracic echocardiography. The method of measurement of the pulmonary vessels diameter is demonstrated in Fig. 4 and the results of measurements are presented in Table II.



Fig. 4. The method of the pulmonary vessels diameter measurement $(1$ - right pulmonary artery, 2 - left pulmonary artery).

TABLE II

The results of the pulmonary vessels diameter measurements.

\begin{tabular}{c|c|c|c|c}
\hline \hline & \multicolumn{2}{|c|}{ Vessels diameters } & \multirow{2}{*}{ Remarks } \\
\cline { 2 - 4 } & $\begin{array}{c}\text { PT } \\
{[\mathrm{cm}]}\end{array}$ & $\begin{array}{c}\text { LPA } \\
{[\mathrm{cm}]}\end{array}$ & $\begin{array}{c}\text { RPA } \\
{[\mathrm{cm}]}\end{array}$ & \\
\hline $\begin{array}{c}\text { patient 1 } \\
\text { (male) }\end{array}$ & 2.93 & 2.10 & 2.40 & $\begin{array}{c}\text { pulmonary } \\
\text { hypertension }\end{array}$ \\
$\begin{array}{c}\text { patient 2 } \\
\text { (male) } \\
\text { patient 3 } \\
\text { female) }\end{array}$ & 1.82 & 0.97 & 1.33 & - \\
\hline
\end{tabular}

To measure the velocity of blood flow through the pulmonary trunk (with a known diameter) the ultrasound Doppler velocimetry was applied. The construction of the developed physical model allowed us to perform the volumetric flow measurement only through the pulmonary arteries (the size of the pulmonary trunk is too small to mounting the flow probe). The construction and load of the pulmonary arteries are similar, therefore volumetric flow through the pulmonary trunk is twice greater than flow measured for one artery. The results of 




Fig. 5. Velocity of flow in human pulmonary trunk and volumetric flow in pulmonary trunk of the physical model.

flow measurements performed on the human and physical model were presented in Fig. 5.

To compare the maximal flow values into the human vessel and the model, the following calculations should be performed. The velocity of blood flow was measured at the centerline of the human pulmonary trunk $(1.83 \mathrm{~cm}$ of diameter), therefore the measured velocity is maximal in the spatial distribution of the flow profile. The diameter of the pulmonary trunk in the model amounts to $2.6 \mathrm{~cm}$. To compare the flow velocities in the patient and model it is necessary to convert the volumetric flow $Q$ measured into the model to the average flow velocity $v_{\mathrm{AV}}$ and recalculate one to the maximal flow velocity $v_{\max }$ in the vessel with other diameter and cross-section area $A_{\text {mod }}$. Assuming the laminar character of flow the maximal blood velocity in the pulmonary trunk of the model is given by the following formula:

$$
v_{\max }=2 v_{\mathrm{AV}}=2 \frac{2 Q}{A_{\bmod }} .
$$

To recalculate this result to equivalent velocity through the pulmonary trunk of a patient, the following equation should be utilized [10]:

$$
v_{1} D_{1}^{2}=v_{2} D_{2}^{2} \text {. }
$$

For the following parameters of the model: the pulmonary trunk diameter $=1.27 \mathrm{~cm}$ and the maximal volumetric flow $=1.65 \mathrm{~L} / \mathrm{min}$, the maximal flow velocity into the centerline of the pulmonary trunk equals $0.46 \mathrm{~m} / \mathrm{s}$. After conversion of this value by ratio $(2.6 / 1.8)^{2}$ calculated basing on formula (2), the maximal flow velocity into the equivalent pulmonary trunk amounts to $0.95 \mathrm{~m} / \mathrm{s}$ $(1 \mathrm{~m} / \mathrm{s}$ for the examined patient).
The result of the first part of verification (the comparison of selected functional parameters of the model with the reference) was presented in Table I.

The second step of verification was to compare the courses of the dilution phenomena occurring in the physical model and the human circulatory system. The comparison was performed for $2.0 \mathrm{~L} / \mathrm{min}$ of cardiac output and $10 \mathrm{~cm}^{3}$, room-temperature indicator. The shapes of indicator dilution curves were presented in Fig. 6 and Fig. 7.

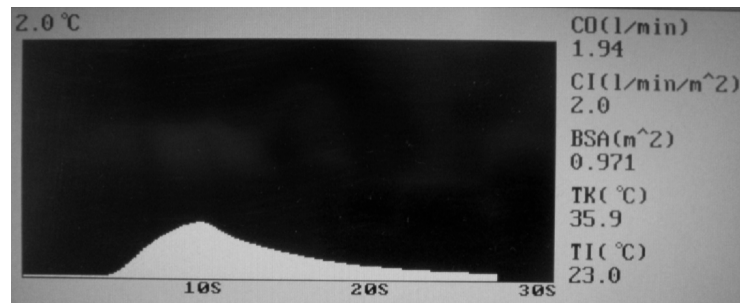

Fig. 6. The indicator dilution curve - investigation on the physical model.

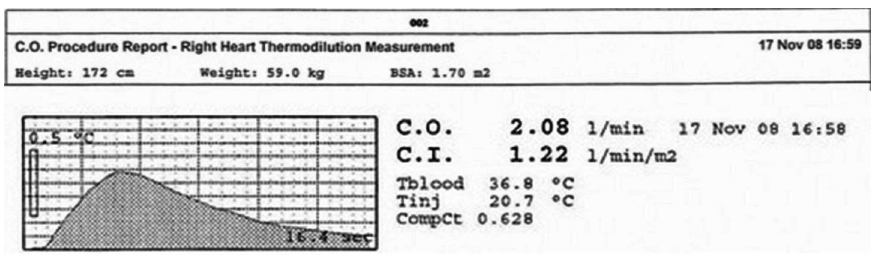

Fig. 7. The indicator dilution curve - examination of the patient.

\section{Discussion}

For correct operation of the developed physical model the liquid level into the equalizing tank and reservoir must be the same. Otherwise, the compensating continuous flow will appear. The simplest way to achieve the equivalences of the liquid levels is to switching off the pneumatic supplying and waiting for the compensation flow fading.

In the physical model the flow oscillations may be observed (see Fig. 5). The oscillations appear at the end of the diastolic phase of the operation cycle. It is caused by two reasons: first, the flexible polyurethane membrane of the ventricle is not supported on the ventricle housing, second, the "hydraulic hammer" effect appears in case of interruption of the flow continuity.

The limitation arising from the mechanical construction of the model is a range of the ejection fraction $(0 \% \ldots 100 \%)$. In the human heart the ejection fraction in physiological state amounts $60 \%$ [11].

The indicator dilution curves registered in the human and model (for cardiac output about $2.0 \mathrm{~L} / \mathrm{min}$ ) are similar. The differences in the measurements time $(16.4 \mathrm{~s}$ for 
the human and $22.5 \mathrm{~s}$ for the model) are arising from the different mathematical algorithms applied in the patient monitors.

\section{Conclusions}

Basing on obtained results, especially on the comparison of the indicator dilution curves, it may be concluded that:

- developed physical model of the pulmonary circulation is able to correct simulation of the dilution phenomenon,

- it may be applied to investigate the cardiac output measurement by means of the thermodilution method.

\section{Acknowledgments}

This work was supported by Polish Ministry of Science (grant No. N N518 336135) and Czesław M. Rodkiewicz Scholarship Foundation.

\section{References}

[1] J.M. Headley, Invasive Hemodynamic Monitoring: Physiological Principles and Clinical Applications, Edwards Lifescience, Irvine 2002.

[2] P.R. Lichtental, Cardiopulmonary Care, Edwards Lifescience, Irvine 2002.

[3] M. Gawlikowski, Ph.D. Thesis, Białystok University of Technology, Białystok 2011 (in Polish).

[4] T. Nishikawa, S. Doshi, Canad. J. Anesthesia 2, 40 (1993).
[5] M. Turowski, Pomiary Automatyka Robotyka 10, 17 (2005) (in Polish).

[6] N. Kothari, Ind. J. Thoracic Cardiovascular Surg. 19, 411 (2003).

[7] L. Mathews, K. Singh, Annals Cardiac Anaesthesia 11, 69 (2008).

[8] J.P. Tournadre, G. Chassard, R. Muchada, Brit. J. Anaesthesia 79, 213 (1997).

[9] K. Klimczak, Clinical Echocardiography, Elselvier Urban and Partner, Wrocław 2008 (in Polish).

[10] A. Szczeklik, M. Tendera, Cardiology, Textbook based on EBM, Vol. 1, Practical Medicine Publisher, Cracow 2009 (in Polish).

[11] W. Ruzyllo, Z. Purzycki, Hemodynamics Diagnostics, PZWL, Warsaw 1984 (in Polish).

[12] J. Gonzalez, Critical Care 7, 153 (2003).

[13] T. Pustelny, P. Struk, Z. Nawrat, M. Gawlikowski, Europ. Phys. J.-Spec. Top. 154, 175 (2007).

[14] M. Gawlikowski, T. Pustelny, B. Przywara-Chowaniec, J. Nowak-Gawlikowska, Acta Phys. Pol. A 118, 1124 (2010).

[15] T. Pustelny, P. Struk, Z. Nawrat, M. Gawlikowski, Europ. Phys. J.-Spec. Top. 154, 171 (2008).

[16] E. Maciak, Z. Opilski, T. Pustelny, M. Bednorz, J. Phys. IV (France) 129, 131 (2005).

[17] M. Gawlikowski, T. Pustelny, B. Przywara-Chowaniec, P. Struk, Acta Phys. Pol. A 114, A81 (2008).

[18] K. Barczak, T. Pustelny, D. Dorosz, J. Dorosz, Europ. Phys. J.-Spec. Top. 154, 11 (2008).

[19] B. Przywara-Chowaniec, L. Polonski, M. Gawlikowski, T. Pustelny, Acta Phys. Pol. A 116, 380 (2009). 